\title{
Asymptotic Modeling of the Thin Film Flow with a Pressure-Dependent Viscosity
}

\author{
Eduard Marušić-Paloka and Igor Pažanin \\ Department of Mathematics, Faculty of Science, University of Zagreb, Bijenička 30, 10000 Zagreb, Croatia \\ Correspondence should be addressed to Igor Pažanin; pazanin@math.hr
}

Received 15 April 2014; Revised 19 June 2014; Accepted 26 June 2014; Published 16 July 2014

Academic Editor: Weiqing Liu

Copyright (C) 2014 E. Marušić-Paloka and I. Pažanin. This is an open access article distributed under the Creative Commons Attribution License, which permits unrestricted use, distribution, and reproduction in any medium, provided the original work is properly cited.

\begin{abstract}
We study the lubrication process with incompressible fluid taking into account the dependence of the viscosity on the pressure. Assuming that the viscosity-pressure relation is given by the well-known Barus law, we derive an effective model using asymptotic analysis with respect to the film thickness. The key idea is to conveniently transform the governing system and then apply two-scale expansion technique.
\end{abstract}

\section{Introduction}

Fluid film bearings are machine elements usually studied in the broader context of tribology. Simply speaking, they consist of two surfaces in relative motion, separated by a thin fluid film, that lubricates the device and minimizes the friction and, consequently, the wear of the device. In our case, the fluid is an incompressible liquid and the two surfaces are rigid. Such elements are very important in mechanical engineering since they provide the reliability of the system and are crucial factor in limiting the dissipation of energy, that is, increasing the efficiency. If a fluid film bearing is well designed, the wear is not an issue, since two surfaces are completely separated by lubricant. It is therefore important to understand the behavior of the fluid film in the bearing. For a systematic treatment of the fundamentals of fluid film lubrication and fluid film bearings we refer the reader to [1].

The foundations of the theoretical treatment of lubrication have been laid already by Rayleigh and Stokes and in particular by the famous work of Reynolds [2]. Assuming that the viscosity is constant and using the thickness as a small parameter, a simple asymptotic approximation can be easily derived providing a well-known Reynolds equation for the pressure of the fluid. However, if the pressures generated in the lubrication flow exceed the range where the viscosity can be considered independent of the pressure, the effects of pressure-dependent viscosity should be taken into account. As reported by numerous researchers (see, e.g., [3-6]), for technological applications in elastohydrodynamic lubrication and in journal bearing applications, where the lubricant is forced through a very narrow gap leading to very high pressures, it is essential to include the variation of the viscosity with pressure. (When considering fluid film lubrication of highly loaded contacts, it is necessary to consider both the viscosity-pressure dependence and the elastic deformation of the surfaces. This case is essential for elastohydrodynamic lubrication. In this paper, for simplicity, we will address the lubrication regime appearing in applications that exhibit pressures high enough to effectively change the lubricant's viscosity, but not so high to initiate significant elastic deformation in the bearing material. Such regime is known as rigidpiezoviscous regime.)

Several models have been used to describe the viscositypressure relation. Barus [7] proposed an exponential law for the viscosity in the form

$$
\mu(p)=\mu_{0} e^{\alpha p} .
$$

Here $\mu_{0}$ represents viscosity at atmospheric pressure while $\alpha>0$ is the pressure-viscosity coefficient. For lubricants, the coefficient $\alpha$ typically varies from 10 to $70 \mathrm{MPa}^{-1}$ (see, e.g., [8]). It is important to emphasize that Barus formula (1) has 
been extensively used in the engineering practice, sometimes combined with temperature dependence.

The exponential law (1) rules out the possibility of deriving the analytical solutions (even for simple flows) and, thus, it has been avoided in theoretical analyses presented in the literature. The other reason lies in the fact that not much has been done in proving the well-posedness of the corresponding boundary-value problems in case of viscosity law (1). Indeed, the existence results for incompressible fluid flows with pressure-dependent viscosity have been provided only under certain technical assumptions on the viscosity which are not fulfilled by the Barus formula. We refer the reader to [9-11]. Recently, however, the first author of the present paper made significant progress on the subject under very general assumption on $\mu(p)$ satisfied by (1) and other empiric laws. First, in [12] he proved the existence and uniqueness of the solution for the stationary Reynolds equation assuming only that the function $p \mapsto \mu(p)$ is of class $C^{1}$ and $\mu>0$ for any value of $p$. After that, in [13], he managed to prove the well-posedness of the stationary Stokes system (describing the real physical problem) assuming that the growth of the function $p \mapsto \mu(p)$ and its derivative is at most exponential:

$$
\begin{array}{r}
0<\mu(p) \leq C_{1} e^{\alpha p}, \quad 0 \leq \mu^{\prime}(p) \leq C_{2} e^{\alpha p}, \\
\left(C_{1}, C_{2}=\text { const. }>0\right) .
\end{array}
$$

It is shown that the corresponding Dirichlet problem has a solution, which is unique under some technical condition which does not rule out the Barus formula. (It is well known that in classical Stokes (or Navier-Stokes) system the pressure is determined only up to constant. To assure uniqueness we need to impose some additional condition, like prescribing the value of its integral over a specified domain. According to [13], it turns out that similar, slightly more technical condition is also needed here to fix the pressure.) For the sake of reader's convenience, we present the key steps of the existence and uniqueness proof in the appendix of this paper.

The result from [13] enables us to investigate the asymptotic behavior of the solution of the lubrication problem with viscosity obeying (1) and that is the goal of this paper. We study the situation appearing naturally in fluid film bearings: two rigid surfaces being in relative motion are separated by a thin layer of fluid; lower surface is assumed to be perfectly smooth, while the upper one is rough with roughness described by some function $h$. Starting from stationary Stokes system with boundary conditions applicable to lubrication flow problem, we perform a rigorous asymptotic analysis with respect to the film thickness. The key idea is to conveniently transform the original problem into the Stokes system with small nonlinear perturbation. As a result, we obtain the effective pressure in the form of the explicit formula clearly acknowledging the viscosity-pressure dependence. To our knowledge, such result cannot be found in the literature and we believe that it could be instrumental for improving the known engineering practice.

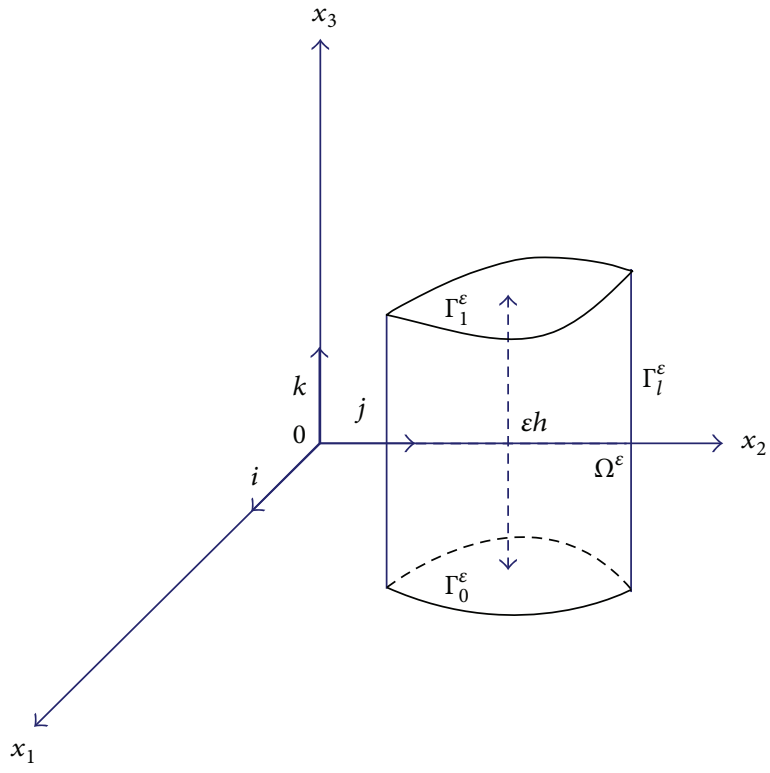

FIgURE 1: The domain.

\section{Description of the Problem}

We consider an incompressible fluid flow in a threedimensional thin domain $\Omega^{\varepsilon}$ defined by

$$
\Omega^{\varepsilon}=\left\{x=\left(x^{\prime}, x_{3}\right) \in \mathbf{R}^{3}: x^{\prime} \in \mathcal{O}, 0<x_{3}<\operatorname{ch}\left(x^{\prime}\right)\right\} .
$$

Here $\mathcal{O} \subset \mathbf{R}^{2}$ is a bounded domain and $h: \overline{\mathcal{O}} \rightarrow\langle 0,+\infty\rangle$ is a given smooth positive function such that $0<h_{0} \leq h\left(x^{\prime}\right) \leq h_{1}$ while $\varepsilon \ll 1$ is a small parameter representing the domain thickness.

We denote by $\Gamma_{0}^{\varepsilon}, \Gamma_{1}^{\varepsilon}$, and $\Gamma_{\ell}^{\varepsilon}$ the lower, the upper, and the lateral boundary of $\Omega^{\varepsilon}$ (see Figure 1); namely,

$$
\begin{aligned}
& \Gamma_{0}^{\varepsilon}=\left\{\left(x^{\prime}, x_{3}\right) \in \mathbf{R}^{3}: x^{\prime} \in \mathcal{O}, x_{3}=0\right\}, \\
& \Gamma_{1}^{\varepsilon}=\left\{\left(x^{\prime}, x_{3}\right) \in \mathbf{R}^{3}: x^{\prime} \in \mathcal{O}, x_{3}=\operatorname{ch}\left(x^{\prime}\right)\right\}, \\
& \Gamma_{\ell}^{\varepsilon}=\left\{\left(x^{\prime}, x_{3}\right) \in \mathbf{R}^{3}: x^{\prime} \in \partial \mathcal{O}, 0<x_{3}<\varepsilon h\left(x^{\prime}\right)\right\} .
\end{aligned}
$$

In view of the application we want to model, we can assume a small Reynolds number and neglect the inertial terms in the governing equations. Thus, we assume that the flow in $\Omega^{\varepsilon}$ is governed by the following system:

$$
\begin{gathered}
-\operatorname{div}\left[2 \mu\left(p^{\varepsilon}\right) \mathbf{D}\left(\mathbf{u}^{\varepsilon}\right)\right]+\nabla p^{\varepsilon}=0 \quad \text { in } \Omega^{\varepsilon}, \\
\operatorname{div} \mathbf{u}^{\varepsilon}=0 \quad \text { in } \Omega^{\varepsilon},
\end{gathered}
$$

where $\mathbf{D}\left(\mathbf{u}^{\varepsilon}\right)=(1 / 2)\left[\nabla \mathbf{u}^{\varepsilon}+\left(\nabla \mathbf{u}^{\varepsilon}\right)^{T}\right]$. The vector field $\mathbf{u}^{\varepsilon}$ denotes the fluid velocity whereas the pressure is given by the scalar field $p^{\varepsilon}$. Throughout the text, the superscript $\varepsilon$ is added in order to stress the dependence of the solution on the small parameter. We assume that the pressure-viscosity dependence is given by the Barus law:

$$
\mu(\xi)=\mu_{0} e^{\alpha \xi}, \quad \mu_{0}, \alpha=\text { const. }>0 .
$$


The choice of boundary conditions highly depends on the devices to be considered. Here we want to study the lubrication process where two rigid surfaces are in relative motion and are separated by a thin layer of fluid. Thus, we impose the following boundary conditions:

$$
\begin{aligned}
& \mathbf{u}^{\varepsilon}=0 \quad \text { on } \Gamma_{1}^{\varepsilon}, \quad \mathbf{u}^{\varepsilon}=\mathbf{w}_{0} \quad \text { on } \Gamma_{0}^{\varepsilon}, \\
& \mathbf{u}^{\varepsilon} \times \mathbf{n}=0 \quad \text { on } \Gamma_{\ell}^{\varepsilon}, \quad p^{\varepsilon}=p_{0} \quad \text { on } \Gamma_{\ell}^{\varepsilon},
\end{aligned}
$$

for given outer pressure $p_{0}$ and constant velocity $\mathbf{w}_{0}$ of relative motion of two surfaces. Note that $\mathbf{w}_{0} \cdot \mathbf{k}=0$ implying $\left.u_{3}^{\varepsilon}\right|_{x_{3}=0}=0$. Throughout the text, $(\mathbf{i}, \mathbf{j}, \mathbf{k})$ will denote the standard Cartesian basis. The unit outward normal on $\Gamma_{\ell}^{\varepsilon}$ is denoted by $\mathbf{n}$.

As mentioned in the Introduction section, the wellposedness of the above problem has been recently established by the first author. For reader's convenience and in order to understand the concept of the solution and its properties, we provide the essential parts of the existence and uniqueness proof in the appendix.

\section{Asymptotic Analysis}

3.1. Transformation Procedure. Our main goal is to find an effective law describing the asymptotic behavior of the flow governed by (5)-(7). Following [14], first we rewrite the governing system by introducing the appropriate transformation for the pressure. Taking into account (6), the momentum equation can be written as

$$
\begin{aligned}
0 & =-\operatorname{div}\left[2 \mu_{0} e^{\alpha p^{\varepsilon}} \mathbf{D}\left(\mathbf{u}^{\varepsilon}\right)\right]+\nabla p^{\varepsilon} \\
& =-\mu_{0} e^{\alpha p^{\varepsilon}} \Delta \mathbf{u}^{\varepsilon}-2 \mu_{0} \alpha e^{\alpha p^{\varepsilon}} \mathbf{D}\left(\mathbf{u}^{\varepsilon}\right) \nabla p^{\varepsilon}+\nabla p^{\varepsilon} .
\end{aligned}
$$

Dividing the above equation by $e^{\alpha p^{\varepsilon}}$ we obtain

$$
-\mu_{0} \Delta \mathbf{u}^{\varepsilon}+e^{-\alpha p^{\varepsilon}} \nabla p^{\varepsilon}=2 \mu_{0} \alpha \mathbf{D}\left(\mathbf{u}^{\varepsilon}\right) \nabla p^{\varepsilon} .
$$

That motivates us to introduce a new function, denoted by $q^{\varepsilon}$, such that

$$
e^{-\alpha p^{\varepsilon}} \nabla p^{\varepsilon}=\nabla q^{\varepsilon}
$$

Obviously, there exists a continuum of such functions given by

$$
q^{\varepsilon}=\frac{1}{\alpha}\left(e^{-\alpha q_{0}}-e^{-\alpha p^{\varepsilon}}\right),
$$

with $q_{0} \in \mathbf{R}$ being arbitrary. The liberty in choice of the parameter $q_{0}$ is crucial for further asymptotic analysis. Noticing that

$$
\nabla p^{\varepsilon}=e^{\alpha p^{\varepsilon}} \nabla q^{\varepsilon}=\frac{1}{e^{-\alpha q_{0}}-\alpha q^{\varepsilon}} \nabla q^{\varepsilon},
$$

equation (9) transforms into

$$
-\mu_{0} \Delta \mathbf{u}^{\varepsilon}+\nabla q^{\varepsilon}=\frac{2 \mu_{0} \alpha}{e^{-\alpha q_{0}-\alpha q^{\varepsilon}}} \mathbf{D}\left(\mathbf{u}^{\varepsilon}\right) \nabla q^{\varepsilon} .
$$

As a result, we arrive at the following transformed system satisfied by $\left(\mathbf{u}^{\varepsilon}, q^{\varepsilon}\right)$ :

$$
\begin{aligned}
&-\mu_{0} \Delta \mathbf{u}^{\varepsilon}+\nabla q^{\varepsilon}= \frac{2 \mu_{0} \alpha}{e^{-\alpha q_{0}}-\alpha q^{\varepsilon}} \mathbf{D}\left(\mathbf{u}^{\varepsilon}\right) \nabla q^{\varepsilon} \quad \text { in } \Omega^{\varepsilon}, \\
& \operatorname{div} \mathbf{u}^{\varepsilon}=0 \quad \text { in } \Omega^{\varepsilon}, \\
& \mathbf{u}^{\varepsilon}=0 \quad \text { on } \Gamma_{1}^{\varepsilon}, \quad \mathbf{u}^{\varepsilon}=\mathbf{w}_{0} \quad \text { on } \Gamma_{0}^{\varepsilon}, \\
& \mathbf{u}^{\varepsilon} \times \mathbf{n}=0 \quad \text { on } \Gamma_{\ell}^{\varepsilon}, \\
& q^{\varepsilon}=\frac{1}{\alpha}\left(e^{-\alpha q_{0}}-e^{-\alpha p_{0}}\right) \quad \text { on } \Gamma_{\ell}^{\varepsilon} .
\end{aligned}
$$

Note that we obtained a nonlinear Stokes-like system, with nonlinearity appearing on the right-hand side in (14) that can be made as small as we need. Indeed, by choosing $q_{0}$ small enough (such assumption is entirely consistent with the existence and uniqueness result provided in the appendix) (i.e., $q_{0}<0$ and $\left|q_{0}\right|$ large enough), we have

$$
\lim _{q_{0} \rightarrow-\infty} \frac{2 \mu_{0} \alpha}{e^{-\alpha q_{0}}-\alpha q^{\varepsilon}}=0 .
$$

Consequently, we will be able to control the nonlinear term $\left(2 \mu_{0} \alpha /\left(e^{-\alpha q_{0}}-\alpha q^{\varepsilon}\right)\right) \mathbf{D}\left(\mathbf{u}^{\varepsilon}\right) \nabla q^{\varepsilon}$ in a way that it does not contribute to the macroscopic model for transformed pressure $q^{\varepsilon}$.

Remark 1. It is important to emphasize that, by choosing $q_{0}$ large and negative, the pressure $q^{\varepsilon}=(1 / \alpha)\left(e^{-\alpha q_{0}}-e^{-\alpha p^{\varepsilon}}\right)$ becomes dominated by the large constant term. However, that is only the transformed pressure (i.e., the pressure satisfying the transformed problem), not the original one. The original pressure $p^{\varepsilon}$, satisfying the governing problem, will remain in starting, physically relevant, range, no matter what value we choose for $q_{0}$. We simply choose the value of the parameter $q_{0}$, construct the asymptotic approximation for $q^{\varepsilon}$ (satisfying transformed Stokes-like problem (14)-(18)), and then reconstruct the approximation for the original pressure from (11). In fact, it can be easily shown by the formal computation that the effective pressure obtained in such way will not depend on $q_{0}$ at all. In view of that, we can conclude that $q_{0}$ can be seen simply as an auxiliary parameter, and, as such, it has no particular physical meaning. Choosing $q_{0}$ large and negative is, indeed, crucial for further asymptotic analysis (coming after the transformation procedure), but it produces no additional constraint to the viscosity law and does not affect the applicability of the main result.

3.2. Approximation for the Transformed Problem. There are several methods that enable us to study the processes in thin domains and to find the asymptotic behavior of the fluid flow. The most sophisticated and precise approach is based on the fine asymptotic analysis with respect to the small parameter $\varepsilon$, representing in our case the film thickness. Such method was first applied in the 50s for deriving, formally, lowerdimensional approximations mostly in theory of lubrication (see [15-17] and the references therein). The method can be roughly described as follows. 
(1) The problem originally posed in thin domain is rewritten on domain with unit thickness by introducing new, dilated (fast) variable. To accomplish that, the differential operator has to be replaced with the new one, containing the derivatives with respect to the fast variable. Consequently, the negative powers of the domain thickness appear, singularly perturbing the operator.

(2) On such, rescaled domain independent of the perturbation parameter $\varepsilon$, one seeks for the unknowns in the form of the asymptotic expansion in powers of $\varepsilon$.

(3) Substituting the expansions in the governing equations and collecting equal powers of $\varepsilon$ lead to the recursive sequence of linear problems yielding the asymptotic model.

We apply the above method to construct the asymptotic approximation for our transformed problem. We introduce the fast variable $y=x_{3} / \varepsilon$ and in the sequel employ the following notation for the partial differential operators:

$$
\begin{gathered}
\nabla_{x^{\prime}} \phi=\frac{\partial \phi}{\partial x_{1}} \mathbf{i}+\frac{\partial \phi}{\partial x_{2}} \mathbf{j}, \quad \Delta_{x^{\prime}} \mathbf{f}=\frac{\partial^{2} \mathbf{f}}{\partial x_{1}^{2}}+\frac{\partial^{2} \mathbf{f}}{\partial x_{2}^{2}}, \\
\operatorname{div}_{x^{\prime}} \mathbf{f}=\frac{\partial f_{1}}{\partial x_{1}}+\frac{\partial f_{2}}{\partial x_{2}}
\end{gathered}
$$

for a scalar function $\phi$ and a vector function $\mathbf{f}=f_{1} \mathbf{i}+f_{2} \mathbf{j}+f_{3} \mathbf{k}$.

We seek for an asymptotic expansion of the unknowns $\mathbf{u}^{\varepsilon}$ and $q^{\varepsilon}$ in the form

$$
\begin{aligned}
& \mathbf{u}^{\varepsilon}=\mathbf{U}^{0}\left(x^{\prime}, y\right)+\varepsilon \mathbf{U}^{1}\left(x^{\prime}, y\right)+\varepsilon^{2} \mathbf{U}^{2}\left(x^{\prime}, y\right)+\cdots, \\
& q^{\varepsilon}=\frac{1}{\varepsilon^{2}} Q^{0}\left(x^{\prime}, y\right)+\frac{1}{\varepsilon} Q^{1}\left(x^{\prime}, y\right)+Q^{2}\left(x^{\prime}, y\right)+\cdots .
\end{aligned}
$$

The leading powers in the expansions for $\mathbf{u}^{\varepsilon}$ and $q^{\varepsilon}$ are suggested by the a priori estimates which can be easily derived from the corresponding weak formulation. Now we substitute the above expansions into the transformed system (14)-(18) and collect the terms with equal powers of $\varepsilon$. Due to (19), from the momentum equation (14) we first deduce

$$
\frac{1}{\varepsilon^{3}}: \quad \frac{\partial Q^{0}}{\partial y}=0
$$

implying $Q^{0}=Q^{0}\left(x^{\prime}\right)$. In view of that, the next term from the momentum equation (14) gives

$$
\frac{1}{\varepsilon^{2}}: \quad-\mu_{0} \frac{\partial^{2} \mathbf{U}^{0}}{\partial y^{2}}+\nabla_{x^{\prime}} Q^{0}+\frac{\partial Q^{1}}{\partial y} \mathbf{k}=0
$$

Taking into account the boundary conditions for the velocity (see (16))

$$
\mathbf{U}^{0}=0 \quad \text { for } y=h, \quad \mathbf{U}^{0}=\mathbf{w}_{0} \quad \text { for } y=0,
$$

we deduce $Q^{1}=Q^{1}\left(x^{\prime}\right)$ and

$$
\begin{array}{r}
\mathbf{U}^{0}\left(x^{\prime}, y\right)=\frac{1}{2 \mu_{0}} y\left(h\left(x^{\prime}\right)-y\right) \mathbf{v}\left(x^{\prime}\right)+\left(1-\frac{y}{h\left(x^{\prime}\right)}\right) \mathbf{w}_{0}, \\
\mathbf{v}=v_{1} \mathbf{i}+v_{2} \mathbf{j} .
\end{array}
$$

Observe that

$$
\mathbf{v}+\nabla_{x^{\prime}} Q^{0}=0
$$

On the other hand, from the divergence equation (15) we have

$$
\frac{1}{\varepsilon}: \quad \operatorname{div}_{x^{\prime}} \mathbf{U}^{0}+\frac{\partial U_{3}^{1}}{\partial y}=0
$$

Integrating from 0 to $h\left(x^{\prime}\right)$ with respect to $y$ yields

$$
\operatorname{div}_{x^{\prime}}\left(\int_{0}^{h} \mathbf{U}^{0} d y\right)=\operatorname{div}_{x^{\prime}}\left(\frac{h^{3}}{12 \mu_{0}} \mathbf{v}+\frac{h}{2} \mathbf{w}_{0}\right)=0 .
$$

As a consequence, we obtain the classical Reynolds equation for $Q^{0}$ :

$$
\operatorname{div}_{x^{\prime}}\left(h^{3} \nabla_{x^{\prime}} Q^{0}\right)=6 \mu_{0} \nabla_{x^{\prime}} h \cdot \mathbf{w}_{0} \quad \text { in } \mathcal{O}
$$

The boundary condition satisfied by $Q^{0}$ reads (see (18))

$$
Q^{0}=0 \quad \text { on } \partial \mathcal{O} \text {. }
$$

Thus, $Q^{0}$ is given as the solution of (29) and (30), being a Dirichlet boundary value problem for linear elliptic equation of second order. The velocity $\mathbf{U}^{0}$ is then determined straightforward from (25) and (26).

3.3. Effective Pressure. So far, we have just constructed an asymptotic approximation $\left(\mathbf{U}^{0}, \varepsilon^{-2} Q^{0}\right)$ for the transformed problem (14)-(18). Now, we have to go back to the original problem and reconstruct the asymptotic solution. In view of (11), we deduce that the pressure distribution has the following form:

$$
P^{0}\left(x^{\prime}\right)=\frac{1}{\alpha} \ln \left(\frac{1}{e^{-\alpha q_{0}}-\left(\alpha / \varepsilon^{2}\right) Q^{0}\left(x^{\prime}\right)}\right), \quad x^{\prime} \in \mathcal{O},
$$

where $Q^{0}$ is given by (29) and (30). The asymptotic approximation for the velocity reads

$$
\begin{array}{r}
\mathbf{u}_{0}^{\varepsilon}(x)=\frac{1}{2 \mu_{0} \varepsilon^{2}} x_{3}\left(\varepsilon h\left(x^{\prime}\right)-x_{3}\right) \mathbf{v}\left(x^{\prime}\right)+\left(1-\frac{x_{3}}{\varepsilon h\left(x^{\prime}\right)}\right) \mathbf{w}_{0}, \\
x \in \Omega^{\varepsilon},
\end{array}
$$

with $\mathbf{v}=-\nabla_{x^{\prime}} Q^{0}$.

From the mathematical point of view, we have to ensure that the effective pressure $P^{0}$ is well defined. Namely, employing classical weak maximum principle combined with the result from [12], we are going to establish the precise upper bound for $q_{0}$ ensuring that the obtained effective pressure is well defined.

From (31) we conclude that the following condition should be fulfilled:

$$
Q^{0}\left(x^{\prime}\right)<\frac{\varepsilon^{2}}{\alpha} e^{-\alpha q_{0}}
$$


If $\nabla_{x^{\prime}} h \cdot \mathbf{w}_{0}<0$, we can simply apply the classical weak maximum principle on (29) and (30) (see Theorem 8.1. from [18]) providing that $Q^{0}\left(x^{\prime}\right) \leq 0$. Condition (33) is then obviously satisfied for any $q_{0} \in \mathbf{R}$. Note that it is not realistic to assume that $\nabla_{x^{\prime}} h \cdot \mathbf{w}_{0}$ does not change sign. In that case we can use the special variant of the maximum principle proved recently in [12]. It yields (see Theorem 1 in [12])

$$
Q^{0}\left(x^{\prime}\right) \leq \mathscr{Z},
$$

where $\mathscr{Z}=\mathscr{E}\left(\mathbf{w}_{0}, h, \mathcal{O}, \mu_{0}\right)$ is given by

$$
\mathscr{Z}=6 \mu_{0} 3^{8 / 5}\left(\frac{3}{2}\right)^{28 / 5} \frac{(2 \pi)^{1 / 4}}{h_{0}^{3}} \operatorname{diam} \mathcal{O}\left|\mathbf{w}_{0}\right||\mathcal{O}|^{8 / 5} .
$$

In view of (34), we deduce that (33) will be satisfied if $\mathscr{Z}<$ $\left(\varepsilon^{2} / \alpha\right) e^{-\alpha q_{0}}$, that is, for

$$
q_{0}<\frac{1}{\alpha} \ln \frac{\varepsilon^{2}}{\alpha \mathscr{Z}} .
$$

It means that, by choosing $q_{0}$ such that (36) holds, we are positive that our effective pressure is well defined.

Remark 2. In [19] a two-dimensional plane flow with a pressure-dependent viscosity obeying Barus law is considered. An approximate system describing the effective flow is formally derived. The idea is to write the governing system in nondimensional form and to estimate the order of magnitude of the unknowns and their derivatives. Under some additional simplifying assumptions concerning this order of magnitudes, a corrected Reynolds equation is directly obtained taking into account the pressure dependence of the lubricant's viscosity. Here we employ different approach. Starting from the original 3D problem, we first construct the asymptotic approximation of the transformed system (14)(18). The transformation procedure is based on the introduction of new unknown (transformed pressure) satisfying (10). Motivated by the recent existence result (see the appendix), we observe that the nonlinear term appearing on the righthand side in the transformed system does not contribute to the macroscopic model leading to a classical Reynolds equation for the transformed pressure. Original pressure, explicitly acknowledging the viscosity-pressure dependence, is then reconstructed using the inverse transformation based on (11). Both results clearly indicate that, in developing lubrication approximation associated to rigid-piezoviscous regime, the pressure dependence of viscosity should be recognized in the process, not a posteriori.

\section{Concluding Remarks}

In this section we give additional important remarks regarding the considered problem and its possible generalizations.

(i) Justification by Error Estimate. In the previous section, we derived an asymptotic model describing the lubrication process with viscosity obeying Barus formula (6). Though the derivation was formal, it provides a good platform for understanding the direct influence of the viscosity-pressure dependence on the effective flow of the lubricant. From the strictly mathematical point of view, formally derived model should be rigorously justified by proving some kind of convergence of the original solution (which we cannot find) towards the asymptotic one. Our aim here is to evaluate the difference between those two solutions in the appropriate norm and to obtain the satisfactory error estimates. By doing that, we validate our formally derived asymptotic model and justify its usage. It is important to notice that the domain $\Omega^{\varepsilon}$ is shrinking so that the integral $L^{r}\left(\Omega^{\varepsilon}\right)$ norms are not appropriate for the error estimate. Indeed, the convergence of $\mathbf{u}^{\varepsilon}-\mathbf{u}_{0}^{\varepsilon}$ to zero in $L^{r}\left(\Omega^{\varepsilon}\right)$ norm does not mean anything since any $L^{\infty}$-bounded function converges to zero in such norm. Therefore, the appropriate convergence is that on the rescaled $\varepsilon$-independent domain. It is well known (see [20-22]) that for the Reynolds pressure $Q^{0}$ the error of approximation (expressed in the rescaled $L^{2}$ norm) is of order $O(\sqrt{\varepsilon})$; namely,

$$
\frac{1}{\sqrt{\left|\Omega^{\varepsilon}\right|}}\left\|\varepsilon^{2} q^{\varepsilon}-Q^{0}\right\|_{L^{2}\left(\Omega^{\varepsilon}\right)} \leq C \sqrt{\varepsilon}
$$

where $\left|\Omega^{\varepsilon}\right|=O(\sqrt{\varepsilon})$ and $C>0$ constant independent of $\varepsilon$. In our case, due to the continuity of the inverse transformation, it immediately follows that for $P^{0}$ the error of approximation is of the same order. For the velocity, we have

$$
\frac{1}{\sqrt{\left|\Omega^{\varepsilon}\right|}}\left\|\mathbf{u}^{\varepsilon}-\mathbf{u}_{0}^{\varepsilon}\right\|_{L^{2}\left(\Omega^{\varepsilon}\right)} \leq C \sqrt{\varepsilon} .
$$

(ii) Correctors for the Transformed Problem. We can continue with the computation and obtain the corrector for the Reynolds model (29) and (30) satisfied by the transformed pressure. More precisely, we can derive second-order model for $\left(\mathbf{v}, Q^{0}\right)$ in the form of Brinkman-type system, as recently proposed by the authors in [23]. However, being very similar to $2 \mathrm{D}$ Navier-Stokes system, it prevents us from applying the above maximum principles in order to deduce that the effective pressure is well defined (as we did for $P^{0}$ ). That will be the subject of our future work.

(iii) General Viscosity-Pressure Relation. Similar approach can be used to extend the analysis to a case of general viscositypressure dependence such that

$$
0<\mu(\xi) \leq C_{1} e^{\alpha \xi}, \quad 0 \leq \mu^{\prime}(\xi) \leq C_{2} e^{\alpha \xi},
$$

where $C_{1}, C_{2}, \alpha=$ const. $>0$. The idea is to introduce $q^{\varepsilon}$ as

$$
B\left(p^{\varepsilon}\right)=\int_{q_{0}}^{p^{\varepsilon}} \frac{d \xi}{\mu(\xi)}
$$

and write the governing system in terms of the new unknown. As a consequence, we will obtain a Stokes-like system with small nonlinear perturbation which can be handled in a similar manner as presented above. For details we refer the reader to [14]. 


\section{Appendix}

\section{The Existence and Uniqueness Result}

Let $\Omega \subset \mathbf{R}^{d}, d=2,3$, be a bounded smooth domain. We assume that the boundary is at least of class $C^{2}$. We consider the following system:

$$
\begin{gathered}
-2 \operatorname{div}[\mu(p) \mathbf{D} \mathbf{u}]+\nabla p=\mathbf{0}, \quad \operatorname{div} \mathbf{u}=0 \quad \text { in } \Omega, \\
\mathbf{u}=\mathbf{g} \quad \text { on } \partial \Omega .
\end{gathered}
$$

We assume that the function $\mathbf{g}$ satisfies the following regularity and compatibility conditions:

$$
\begin{gathered}
\mathbf{g} \in W^{2-1 / \beta, \beta}(\Omega)^{d}, \quad \text { for some } \beta>d \\
\int_{\partial \Omega} \mathbf{g} \cdot \mathbf{n}=0
\end{gathered}
$$

For the dependence of the viscosity on the pressure we assume that $p \mapsto \mu(p)$ is given by the Barus law

$$
\mu(p)=\mu_{0} e^{\alpha p} .
$$

In the following we prove that the above problem has a solution, which is unique, under some technical conditions. In standard Stokes (or Navier-Stokes) system the pressure is obviously determined only up to a constant, so it is not unique unless we impose some additional condition, like prescribing the value of its integral over $\Omega$ or prescribing the value of the pressure in some point of the domain $\Omega$. That is less obvious here, since the pressure appears in the viscosity formula. However, it turns out that similar condition is needed here to fix the pressure. Note that we prescribe velocity on the whole boundary $\partial \Omega$. That is just to be able to clearly present the main ideas of the existence and uniqueness proof by avoiding unnecessary notational confusion. In the governing system (5)-(7) we prescribed pressure on the lateral part of the boundary in order to treat the situation naturally arising in the applications. Since we neglected the inertial term in the momentum equation, prescribing pressure boundary condition does not represent a serious obstacle (see, e.g., [24]), so the adaptation of the presented proof is just technical, but straightforward.

Theorem A.1 (existence result). Let g satisfy (A.2). Assume in addition that (A.3) holds. Then the problem (A.1) has a solution $(\mathbf{u}, p) \in \mathbf{X}=W^{2, \beta}(\Omega)^{d} \times W^{1, \beta}(\Omega)$.

Before proving the above assertion let us conveniently transform the governing system. For some $\sigma \in \mathbf{R}$ we define two mappings. The first one is

$$
B(p, \sigma)=\int_{\sigma}^{p} \frac{d s}{\mu(s)}=\frac{1}{\alpha \mu_{0}}\left(e^{-\alpha \sigma}-e^{-\alpha p}\right) .
$$

$B$ is of class $C^{1}$ and the function $B(\cdot, \sigma): \mathbf{R} \mapsto \mathbf{R}$ isstrictly increasing (and thus injective), for any parameter $\sigma$.
Furthermore $\operatorname{Im} B(\cdot, \sigma)=\left[M_{\sigma}^{-}, M_{\sigma}^{+}\right]$, where $M_{\sigma}^{+}=$ $\lim _{p \rightarrow+\infty} B(p, \sigma)=e^{-\alpha \sigma} / \alpha \mu_{0}$ and $M_{\sigma}^{-}=\lim _{p \rightarrow+\infty} B(p, \sigma)=$ $-\infty$. We can now define the second one as

$$
H(\cdot, \sigma)=B^{-1}(\cdot, \sigma) \Longrightarrow H(q, \sigma)=\frac{1}{\alpha} \ln \left(\frac{1}{e^{-\alpha \sigma}-\alpha \mu_{0} q}\right) \text {. }
$$

Thus $q \mapsto H(q, \sigma)$ is an inverse of the function $p \mapsto B(q, \sigma)$, while $\sigma$ is treated only as a parameter. Obviously $H(\cdot, \sigma)$ : $\left[M_{\sigma}^{-}, M_{\sigma}^{+}\right] \rightarrow \mathbf{R}$ is well defined, strictly increasing, and smooth. Furthermore $(\partial H / \partial q)(q, \sigma)=\mu(p)=\mu(H(q, \sigma))$. Next we define the function

$$
b(q, \sigma)=\mu^{\prime}(p)=\mu^{\prime}(H(q, \sigma))=\frac{\alpha \mu}{e^{-\alpha \sigma}-\alpha \mu_{0} q}
$$

that we need in the sequel. It is defined only on $\left[M_{\sigma}^{-}, M_{\sigma}^{+}\right]$and continuous. Using the assumptions on $\mu$ it is an easy exercise to prove the following technical result.

Lemma A.2. Let $\mu: \mathbf{R} \rightarrow \mathbf{R}$ satisfy the Barus law (A.3). Then

$$
\lim _{\sigma \rightarrow-\infty} b(q, \sigma)=0, \quad \text { for any } q \in \mathbf{R} \text {. }
$$

We now define the new unknown

$$
q=B(p, \sigma) .
$$

We can now rewrite our system in terms of that new unknown and it reads

$$
\begin{gathered}
-\Delta \mathbf{u}+\nabla q=b(q, \sigma) \mathbf{D u} \nabla q, \quad \operatorname{div} \mathbf{u}=0 \quad \text { in } \Omega, \\
\mathbf{u}=\mathbf{g} \quad \text { on } \partial \Omega .
\end{gathered}
$$

Proof of Theorem A.1. The idea is to construct the sequence $\left(\mathbf{u}_{n}, q_{n}\right)$ by taking $\mathbf{u}_{1}=0, q_{1}=0$ and for $n>1$

$$
\begin{gathered}
-\Delta \mathbf{u}_{n}+\nabla q_{n}=b\left(q_{n-1}, \sigma\right) \mathbf{D} \mathbf{u}_{n-1} \nabla q_{n-1} \quad \text { in } \Omega, \\
\operatorname{div} \mathbf{u}_{n}=0 \quad \text { in } \Omega, \quad \mathbf{u}_{n}=\mathbf{g} \quad \text { on } \partial \Omega .
\end{gathered}
$$

The classical result by Cattabriga [25] implies for any $\beta>d$ and any $\left(\mathbf{u}_{n-1}, q_{n-1}\right) \in W^{2, \beta}(\Omega)^{d} \times W^{1, \beta}(\Omega)$ the existence of such $\left(\mathbf{u}_{n}, q_{n}\right) \in W^{2, \beta}(\Omega) \times W^{1, \beta}(\Omega)$. Furthermore, for some $C_{\beta}>0$

$$
\begin{aligned}
& \left|\mathbf{u}_{n}\right|_{W^{2, \beta}(\Omega)}+\left|q_{n}\right|_{W^{1, \beta}(\Omega)} \\
& \quad \leq C_{\beta}\left(\left|b\left(q_{n-1}, \sigma\right) \mathbf{D} \mathbf{u}_{n-1} \nabla q_{n-1}\right|_{L^{\beta}(\Omega)}+|\mathbf{g}|_{W^{2-1 / \beta, \beta}(\partial \Omega)}\right) .
\end{aligned}
$$

If, for given $\lambda \in \mathbf{R}$ and $x_{0} \in \Omega$, we impose

$$
q_{n}\left(x_{0}\right)=\lambda
$$

then the solution is unique. Furthermore, assuming that

$$
\begin{gathered}
\left|\mathbf{u}_{n-1}\right|_{W^{2, \beta}(\Omega)} \leq M \\
\left|q_{n-1}\right|_{W^{1, \beta}(\Omega)} \leq M, \quad q_{n-1}\left(x_{0}\right)=\lambda
\end{gathered}
$$


and denoting $C(\beta, \infty)$ as the imbedding constant $W^{1, \beta}(\Omega) \subset$ $L^{\infty}(\Omega)$, we obtain

$$
\begin{aligned}
& \left|q_{n}\right|_{W^{1, \beta}(\Omega)} \\
& \leq C_{\beta}\left(\left|b\left(q_{n-1}, \sigma\right)\right|_{L^{\infty}(\Omega)}\left|\mathbf{D} \mathbf{u}_{n-1}\right|_{L^{\infty}(\Omega)}\left|\nabla q_{n-1}\right|_{L^{\beta}(\Omega)}\right. \\
& \left.\quad+|\mathbf{g}|_{W^{2-1 / \beta, \beta}(\partial \Omega)}\right) \\
& \leq C_{\beta}\left(M^{2} C(\beta, \infty)^{2}\left|b\left(q_{n-1}, \sigma\right)\right|_{L^{\infty}(\Omega)}+|\mathbf{g}|_{W^{2-1 / \beta, \beta}(\partial \Omega)}\right) .
\end{aligned}
$$

For any $x \in \bar{\Omega},\left|q_{n-1}(x)\right| \leq C(\beta, \infty) M \equiv \bar{M}$. For any $\sigma$ such that $e^{-\alpha \sigma}>\alpha \mu_{0} \bar{M}$, we have

$$
b\left(q_{n-1}(x), \sigma\right)=\frac{\alpha \mu}{e^{-\alpha \sigma}-\alpha \mu_{0} q_{n-1}(x)}<\frac{\alpha \mu}{e^{-\alpha \sigma}-\alpha \mu_{0} \bar{M}} .
$$

Thus, for any $x \in \bar{\Omega}$ and $n \in \mathbf{N}$ and for any $\varepsilon>0$, there exists $\sigma_{0}$ such that for any $\sigma<\sigma_{0}$

$$
\left|b\left(q_{n-1}(x), \sigma\right)\right|_{L^{\infty}(\Omega)} \leq \frac{\varepsilon}{C_{\beta} M^{2} C(\beta, \infty)^{2}} .
$$

It is important to notice that $\sigma_{0}$ does not depend neither on $x$ nor on $n$. For $G=|\mathbf{g}|_{W^{2-1 / \beta, \beta}(\partial \Omega)}$ we choose $M=2 C_{\beta} G$ and for $\varepsilon<G$ (A.13) and (A.14) imply that

$$
\left|\mathbf{u}_{n}\right|_{W^{2, \beta}(\Omega)} \leq M, \quad\left|q_{n}\right|_{W^{1, \beta}(\Omega)} \leq M .
$$

That proves the boundness of the sequence $\left(\mathbf{u}_{n}, q_{n}\right)$. Then, up to a subsequence,

$$
\begin{aligned}
& \mathbf{u}_{n} \rightarrow \mathbf{u} \quad \text { weakly in } W^{2, \beta}(\Omega)^{d}, \\
& q_{n} \rightarrow q \quad \text { weakly in } W^{1, \beta}(\Omega) .
\end{aligned}
$$

Compact embedding $W^{1, \beta}(\Omega) \subset C(\bar{\Omega})$ and (A.17) implies the strong convergence

$$
\begin{gathered}
\mathbf{u}_{n} \longrightarrow \mathbf{u} \quad \text { in } W^{1, \sigma}(\Omega)^{d}, \quad \sigma<\frac{d \beta}{d-\beta} \text { in } C^{1}(\bar{\Omega})^{d}, \\
q_{n} \longrightarrow q \quad \text { in } L^{\sigma}(\Omega) \quad \text { in } C(\bar{\Omega}) .
\end{gathered}
$$

That is enough to pass to the limit in (A.10) and to prove the existence of solution of the transformed system (A.9). Furthermore,

$$
|\mathbf{u}|_{W^{2, \beta}(\Omega)} \leq M, \quad|q|_{W^{1, \beta}(\Omega)} \leq M
$$

Due to (A.12) we have in addition $q\left(x_{0}\right)=\lambda$. To prove that $(\mathbf{u}, p), p=H(q, \sigma)$, with $H$ defined by (A.5), is the solution to the original system (A.1), we need to verify that $q(x)$ is in the range of function $H(\cdot, \sigma), \operatorname{Im} H(\cdot, \sigma)=\left\langle-\infty, M_{\sigma}^{+}\right]$, for some $\sigma<0$. We thus need to verify that $q(x) \leq M_{\sigma}^{+}=e^{-\alpha \sigma} / \alpha \mu_{0}$, $\forall x \in \Omega$, which is fulfilled (see Lemma A.2) for sufficiently large negative $\sigma$. Indeed, we know that $q(x) \leq \bar{M}$. It is therefore sufficient to choose $\sigma$ such that $M_{\sigma}^{+} \geq \bar{M}$.
Theorem A.3 (uniqueness result). Assume that the viscosity function satisfies the Barus law (A.3). Let $(\mathbf{u}, p) \in \mathbf{X}=$ $W^{2, \beta}(\Omega)^{d} \times W^{1, \beta}(\Omega)$ be the solution to the problem (A.1) whose existence is guaranteed by Theorem A.1. If in addition we prescribe the value of the pressure in some point $x_{0} \in \Omega$, that is, for some $\kappa \in \mathbf{R}$, we impose

$$
p\left(x_{0}\right)=\kappa
$$

then there are no other solutions in $\mathbf{X}$.

Proof. Suppose that the problem has two solutions $(\mathbf{u}, p),(\mathbf{w}, \pi)$ in $\mathbf{X}$. We apply the transformation procedure as in the existence proof and take $q=B(p, \sigma), \eta=B(\pi, \sigma)$. We denote by $\mathbf{E}=\mathbf{u}-\mathbf{w}, e=q-\eta$. Then, obviously $\mathbf{E}=0$ on $\partial \Omega$ and the difference $(\mathbf{E}, e)$ satisfies the Stokes system

$$
-\Delta \mathbf{E}+\nabla e=b(q, \sigma) \nabla q D \mathbf{u}-b(\eta, \sigma) \nabla \eta D \mathbf{w} \equiv \mathscr{R} .
$$

Thus, the standard a priori estimate (see, e.g., [25]) implies

$$
|\mathbf{E}|_{W^{2, \beta}(\Omega)}+|e|_{W^{1, \beta}(\Omega) / \mathbf{R}} \leq C_{\beta}|\mathscr{R}|_{L^{\beta}(\Omega)} .
$$

On the other hand, due to (A.6), (A.7), for given $\varepsilon>0$ we can choose $\sigma$ in definition of $b$ such that

$$
\begin{aligned}
& |b(q, \sigma) \nabla q D \mathbf{u}|_{L^{\beta}(\Omega)} \leq \frac{\varepsilon}{2 C_{\beta}} \\
& |b(\eta, \sigma) \nabla \eta D \mathbf{w}|_{L^{\beta}(\Omega)} \leq \frac{\varepsilon}{2 C_{\beta}}
\end{aligned}
$$

and thus $|\mathbf{E}|_{W^{2, \beta}(\Omega)}+|e|_{W^{1, \beta}(\Omega) / \mathbf{R}} \leq \varepsilon$. As $\varepsilon$ was arbitrary, we conclude that $\mathbf{E}=0$ and $e=$ const. That proves the uniqueness of the velocity since it is independent of choice of $\sigma$. On the other hand, $q$ and $\eta$ do depend on $\sigma$. However, their difference does not since

$$
e(x)=q(x)-\eta(x)=\frac{1}{\alpha \mu_{0}}\left(e^{-\alpha \pi(x)}-e^{-\alpha p(x)}\right) .
$$

Then, if we prescribe the value of the pressure in some point $x_{0} \in \Omega$, that is, if for given $\kappa \in \mathbf{R}$ we put $p\left(x_{0}\right)=\pi\left(x_{0}\right)=$ $\kappa$, we have $e=0$ so that $e^{-\alpha \pi(x)}=e^{-\alpha p(x)}$ implying $p(x)=$ $\pi(x)$.

\section{Conflict of Interests}

The authors declare that there is no conflict of interests regarding the publication of this paper.

\section{Acknowledgments}

This research was supported by the Ministry of Science, Education and Sports, Croatia, Grant 037-0372787-2797. The authors would like to thank the referees for valuable comments and suggestions that helped to correct and improve their paper. 


\section{References}

[1] A. Z. Szeri, Fluid Film Lubrication: Theory and Design, Cambridge University Press, 1998.

[2] O. Reynolds, "On the theory of lubrication and its applications to Mr. Beauchamp Towers experiments, including an experimental determination of the viscosity of olive oil," Philosophical Transactions of the Royal Society of London, vol. 177, pp. 157-234, 1886.

[3] W. R. Jones, "Pressure viscosity measurement for several lubricants," ASLE Transactions, vol. 18, no. 4, pp. 249-262, 1975.

[4] S. C. Jain, R. Sinhasan, and D. V. Singh, "Consideration of viscosity variation in determining the performance characteristics of circular bearings in the laminar and turbulent regimes," Wear, vol. 86, no. 2, pp. 233-245, 1983.

[5] D. R. Gwynllyw, A. R. Davies, and T. N. Phillips, "On the effects of a piezoviscous lubricant on the dynamics of a journal bearing," Journal of Rheology, vol. 40, no. 6, pp. 1239-1266, 1996.

[6] A. Goubert, J. Vermant, P. Moldenaers, A. Göttfert, and B. Ernst, "Comparison of measurement techniques for evaluating the pressure dependence of the viscosity," Applied Rheology, vol. 11, no. 1, pp. 26-37, 2001.

[7] C. Barus, "Isothermals, isopiestics and isometrics relative to viscosity," The American Journal of Science, vol. 45, pp. 87-96, 1893.

[8] P. A. Kottke, Rheological implications of tension in liquids [Ph.D. thesis], Georgia Institute of Technology, 2004.

[9] M. Renardy, "Some remarks on the Navier-Stokes equations with a pressure-dependent viscosity," Communications in Partial Differential Equations, vol. 11, no. 7, pp. 779-793, 1986.

[10] F. Gazzola, "A note on the evolution Navier-Stokes equations with a pressure-dependent viscosity," Zeitschrift für Angewandte Mathematik und Physik, vol. 48, no. 5, pp. 760-773, 1997.

[11] J. Málek, J. Nečas, and K. R. Rajagopal, "Global analysis of the flows of fluids with pressure-dependent viscosities," Archive for Rational Mechanics and Analysis, vol. 165, no. 3, pp. 243-269, 2002.

[12] E. Marušić-Paloka and S. Marušić, "Analysis of the Reynolds equation for lubrication in case of pressure-dependent viscosity," Mathematical Problems in Engineering, vol. 2013, Article ID 710214, 10 pages, 2013.

[13] E. Marusic-Paloka, "An analysis of the Stokes system with pressure dependent viscosity," In press.

[14] E. Marušić-Paloka and I. Pažanin, "A note on the pipe flow with a pressure-dependent viscosity," Journal of Non-Newtonian Fluid Mechanics, vol. 197, pp. 5-10, 2013.

[15] M. van Dyke, Perturbation Methods in Fluid Mechanics, The Parabolic Press, Stanford, Calif, USA, 1975.

[16] G. H. Wannier, "A contribution to the hydrodynamics of lubrication," Quarterly of Applied Mathematics, vol. 8, pp. 1-32, 1950.

[17] R. K. Zeytouninan, Modelisation Asymptotique en Mecanique des Fluides Newtoniens, Collection SMAI, Springer, Berlin, Germany, 1994.

[18] D. Gilbarg and N. S. Trudinger, Elliptic Partial Differential Equations of Second Order, Springer, 1997.

[19] K. R. Rajagopal and A. Z. Szeri, "On an inconsistency in the derivation of the equations of elastohydrodynamic lubrication," Proceedings of the Royal Society of London A, vol. 459, no. 2039, pp. 2771-2786, 2003.
[20] S. A. Nazarov, "Asymptotic solution of the Navier-Stokes problem on the flow of a thin layer of fluid," Siberian Mathematical Journal, vol. 31, no. 2, pp. 296-307, 1990.

[21] A. Duvnjak and E. Marusic-Paloka, "Derivation of the Reynolds equation for lubrication of a rotating shaft," Archivum Mathematicum, vol. 36, no. 4, pp. 239-253, 2000.

[22] J. Wilkening, "Practical error estimates for Reynolds' lubrication approximation and its higher order corrections," SIAM Journal on Mathematical Analysis, vol. 41, no. 2, pp. 588-630, 2009.

[23] E. Marušić-Paloka, I. Pažanin, and S. Marušić, "Second order model in fluid film lubrication," Comptes Rendus: Mecanique, vol. 340, no. 8, pp. 596-601, 2012.

[24] C. Conca, F. Murat, and O. Pironneau, "The Stokes and Navier-Stokes equations with boundary conditions involving the pressure," Japanese Journal of Mathematics, vol. 20, pp. 263318, 1994.

[25] L. Cattabriga, "Su un problema al contorno relativo al sistema di equazioni di Stokes," Rendiconti del Seminario Matematico della Università di Padova, vol. 31, pp. 308-340, 1961. 


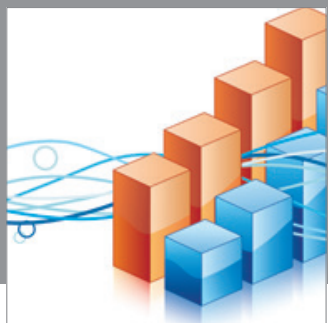

Advances in

Operations Research

mansans

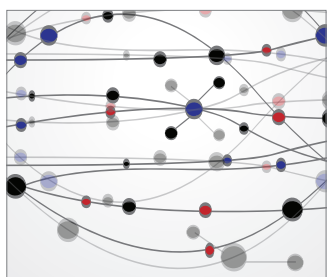

The Scientific World Journal
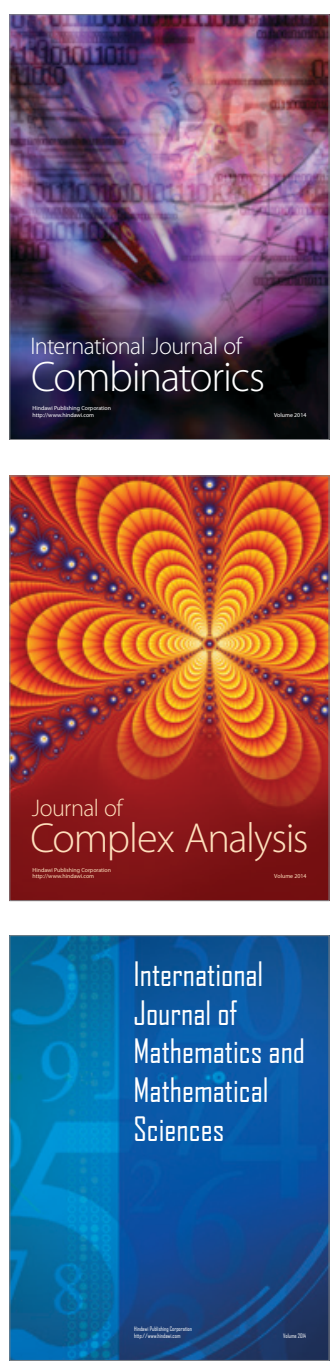
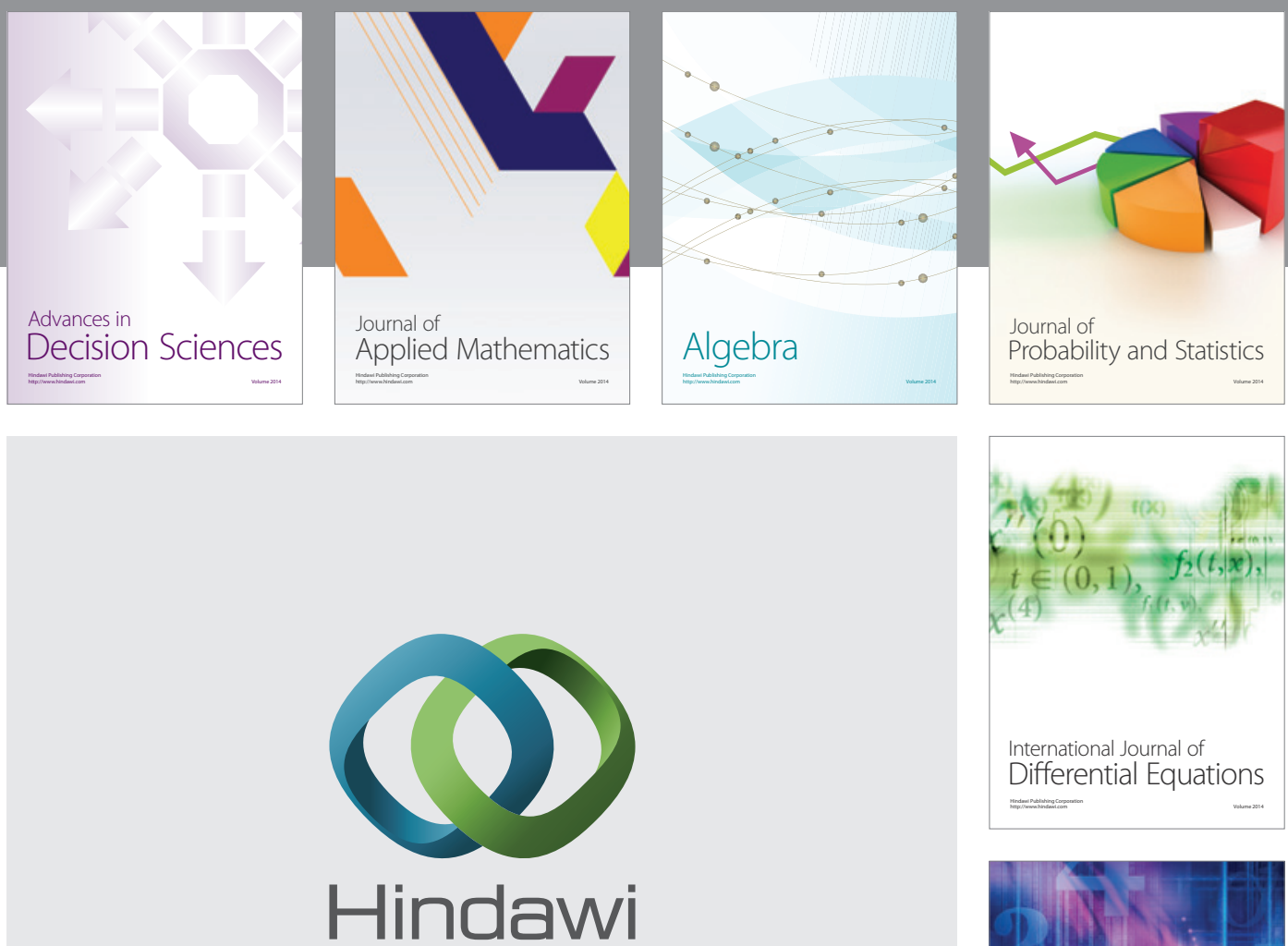

Submit your manuscripts at http://www.hindawi.com
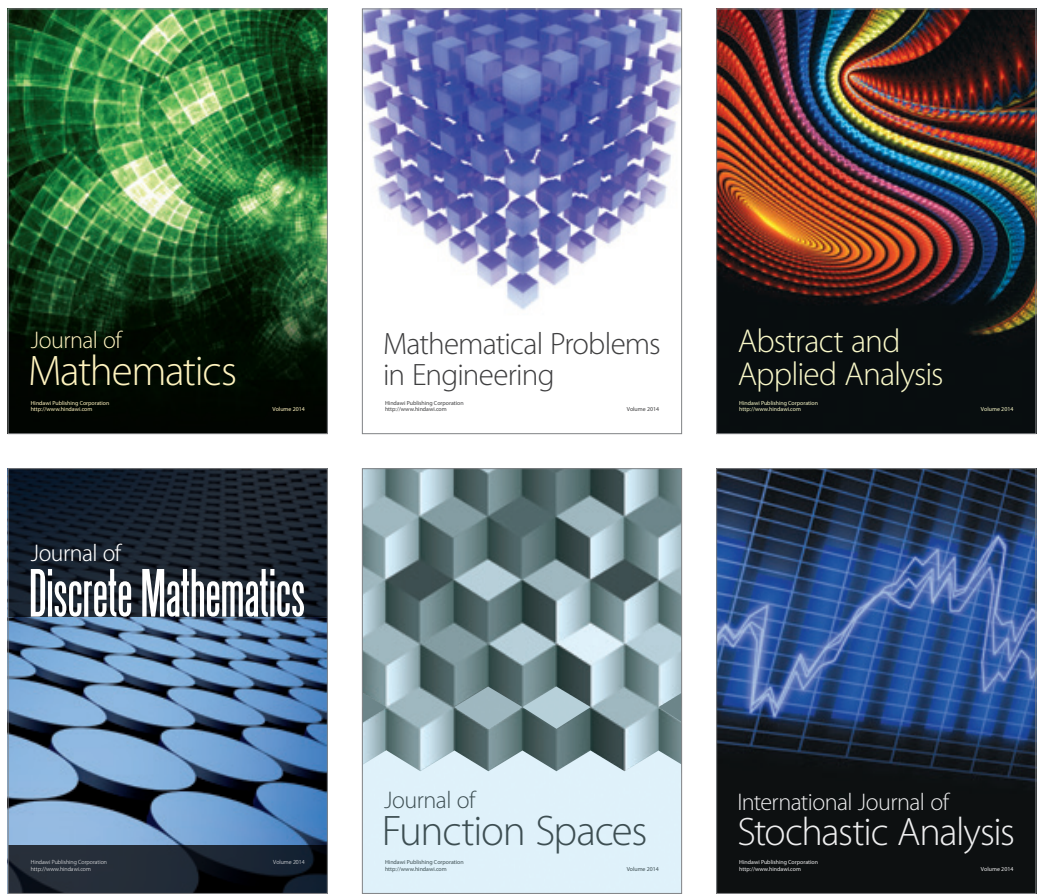

Journal of

Function Spaces

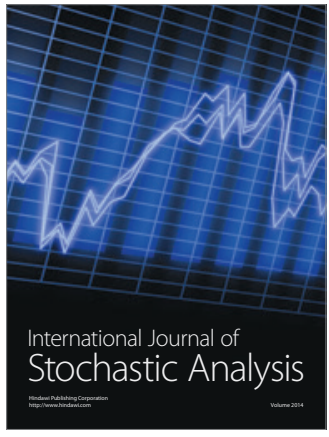

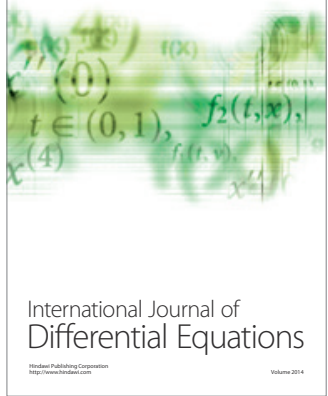
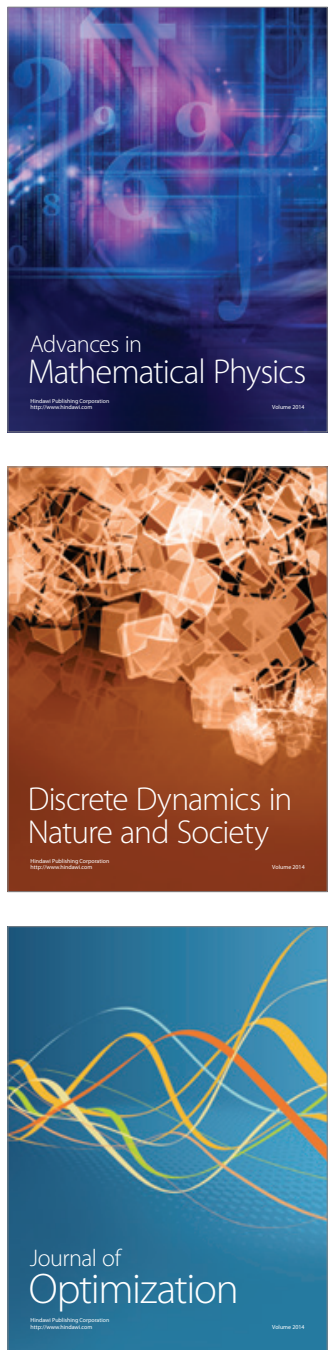